Desi gn, construction, and perfor mance of a composite mirror for coll ecting Thomson scattered I ight fromthe I arge hel ical device pl asma

\begin{tabular}{|c|c|}
\hline $\begin{array}{l}\text { j our nal or } \\
\text { publ i cat i on title }\end{array}$ & Revi ew of Sci ent i i c I nst ruments \\
\hline vol une & Vol . 75 \\
\hline number & I ssue10 \\
\hline page $r$ ange & pp. $3878-3880$ \\
\hline year & $200410-01$ \\
\hline URL & ht t p: //hdl . handl e. net /10655/2286 \\
\hline
\end{tabular}




\title{
Design, construction, and performance of a composite mirror for collecting Thomson scattered light from the large helical device plasma
}

\author{
K. Narihara, I. Yamada, H. Hayashi, and K. Yamauchi \\ National Institute for Fusion Science, Toki 592-5292, Japan
}

(Presented on 20 April 2004; published 7 October 2004)

\begin{abstract}
A $1.5 \mathrm{~m} \times 1.8 \mathrm{~m}$ rectangular composite mirror composed of 138 segment hexagonal spherical mirrors was constructed for collecting Thomson scattered light from the Large Helical Device plasma. The hexagonal mirrors with side length of $87 \mathrm{~mm}$ were patched on the surface of a framework made of glass fiber reinforced plastic. The position and angular orientation of each mirror are adjusted with three pairs of push-and-pull screws attached to the back plane of the mirrors so that the image of a tiny light source $(0.1 \mathrm{~mm}$ in diameter) formed by each segment mirror be minimized and coincide with each other on a charge coupled device plate. The optical quality and its long-term stability of the assembled mirror have been monitored and sufficient for the present purpose. (C) 2004 American Institute of Physics. [DOI: 10.1063/1.1787919]
\end{abstract}

\section{INTRODUCTION}

Incoherent Thomson scattering (TS) of intense laser off plasma electrons is a well-established method to measure the electron temperature $\left(T_{e}\right)$ and density $\left(n_{e}\right)$ in the contemporary fusion plasma experiment. The cross section for TS, $\sigma \sim$ (classical electron radius) $^{2} \sim 8 \times 10^{-24} \mathrm{~cm}^{2}, \quad$ and the typical $n_{e}$ of the fusion-oriented plasma experiments, $n_{e} \sim 10^{14} \mathrm{~cm}^{-3}$, give the mean free path of TS scattering $1=1 /\left(\sigma n_{e}\right) \sim 10^{11} \mathrm{~cm}$, which is much larger than the size of laboratory plasmas. Therefore, in order to obtain scattering signals intense enough to reduce $T_{e}$ and $n_{e}$ accurately, we must prepare a very intense laser, bright optics, and highly efficient detectors. In practice, the intensity of laser is usually limited by the laser-induced damage or unfavorable thermal effects on the optical components, and the efficiency of the detectors commonly used for TS seems to have already reached their matured levels. On the other hand, we can easily raise the brightness (speed) of the optics by making a solid angle of light gathering optics as large as possible. Thus we designed a TS system which adopts a large rectangular view window of $0.33 \mathrm{~m} \times 0.58 \mathrm{~m}$ in the effective area and a large mirror $(1.5 \mathrm{~m} \times 1.8 \mathrm{~m})$ as a light collecting optic for the Large Helical Device (LHD). ${ }^{1}$ It is not easy to fabricate a mirror of this size and shape as one body; even if it is possible it would be very expensive. To alleviate this technical difficulty we adopted a composite mirror composed of many segment mirrors patched on a framework of the desired size and shape. In this article, we describe the design, assembly, and performance of this composite mirror.

\section{DESIGN CONSIDERATIONS}

The TS installed on LHD adopts a backward scattering configuration with the laser window and the viewing window on the same vertical port (4-O). ${ }^{2}$ The laser beam goes through a quartz window attached at the center of 4-O port so that it runs along a major radius on the midplane $(z=0$ plane). The position, shape, and inclination of the observa- tion window were determined so that the whole image of the laser beam in the plasma can be observed with highest spatial resolution and with largest solid angle. The center of the mirror's curvature (CMC) is located near the center of the window, which minimized all but spherical aberrations. In practice, CMC is $2 \mathrm{~cm}$ apart from the surface of the window in the air side, which facilitates an easy alignment of the segment mirrors. The linear size of the composite mirror is proportional to the radius of the mirror's curvature $\left(R_{c}\right)$. In view of cost, the smaller $R_{c}$ is favorable, which, however, introduces larger spherical aberration, necessitating optical fiber with larger diameter to accept the focused light and degrading the spatial resolution. We chose to optimize $R_{c}$ $=4.5 \mathrm{~m}$ and a rectangular mirror of $1.5 \mathrm{~m} \times 1.8 \mathrm{~m}$.

The mirror was to be set at $R=12 \mathrm{~m}$ from the center of the LHD with its side inclined $30^{\circ}$ from the vertical line. The LHD building is air conditioned so that the room temperature variation is usually within a few degrees. However, very rare electric power stops will cause temperature change larger than $10^{\circ}$. Though very slow rise and fall of electric currents in the superconducting (SC) coils before and after daily experiments would exert no appreciable force on metallic material around LHD, the future-planned fast coil current drive during plasma discharge or occasional partial quenches in SC current would cause eddy currents and exert considerable force on the metallic material around LHD. Earth's gravity is also acting on the mirror. In the above environmental condition, the segment mirrors should direct to the common focal point stably to give accurate $n_{e}$ profile for up to ten years. Considering the possibilities that the magnetic field and the room temperature change occasionally, we chose glass fiber reinforced plastic (GFRP) as material for the framework, which has a high electric resistivity, small linear thermal expansion coefficient, and high strength.

The number of the segment mirrors, $N_{m}$, is a key parameter in designing a composite mirror. Let us suppose a set of segment mirrors, which covers the area of $1.5 \mathrm{~m} \times 1.8 \mathrm{~m}$, has the mean $R_{c}=4.5 \mathrm{~m}$ and its standard deviation 

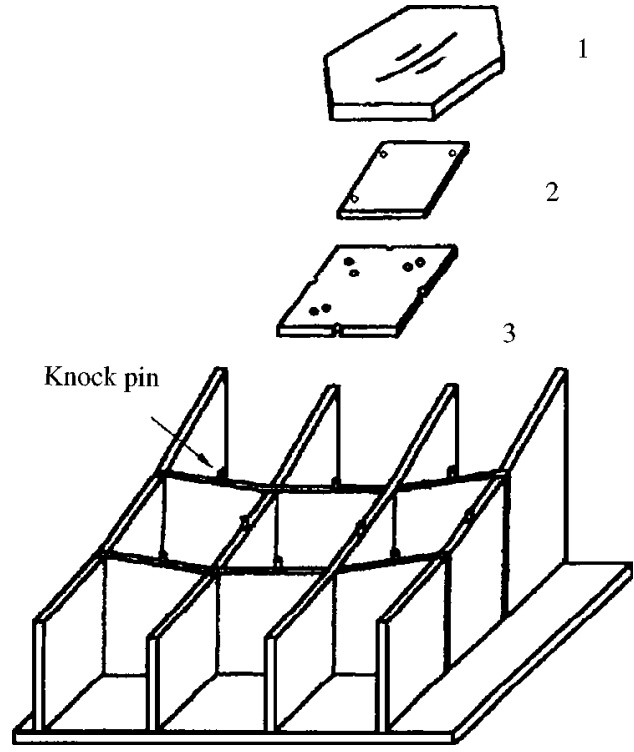

FIG. 1. Components of the composite mirror. From the bottom: framework; adjustment plate (C plate), back plate (B plate), and segment mirror.

$\delta R_{c} \sim 2 \mathrm{~mm}$, and consider the cases of the larger and smaller $N_{m}$. As easily imaged, the smaller $N_{m}$ will make the assembled mirror thicker, heavier, and therefore more expensive; furthermore, it will introduce a larger rms deviation from an ideal spherical surface. Conversely, the larger $N_{m}$ scheme, which uses many smaller size segment mirrors, will encounter a difficulty of preparing a large number of mirrors with $R_{c} \sim 4.5 \mathrm{~m}$ and $\delta R_{c}<2 \mathrm{~mm}$, the fabrication of such being much easier for a larger size mirror. Balancing the positive and negative factors involved, we chose $N_{m}=138$, which gives a rough optimization in the optical performance and the construction cost.

In order to get the highest rigidity with a minimum amount of material weight, we adopted a "honeycomb" structure for the framework, a part of which is schematically shown in Fig. 1, by bonding 14 different 10-mm-thick GFRP planes together. On the hollow side, we patched 138 adjustment GFRP planes (C planes) of $10 \mathrm{~mm}$ in thickness with three threaded holes for pushing and three through holes for pulling it. Four pins set at four sides guaranteed precise positioning. On the back of the honeycomb we bonded a 10 -mm-thick GFRP plane with 138 holes of $100 \mathrm{~mm}$ in diameter for accessing the adjustment screws.

The basic segment mirrors are 2 -cm-thick hexagon with a side length of $87 \mathrm{~mm}$. A plane surface can be patched by these hexagons without a gap, but as the surface's curvature becomes significant as in the present case the gaps among the mirrors become wider, lowering the effective reflectivity of the assembled mirror. To alleviate this problem, the basic shape is modified into 11 types $(\mathrm{A}-\mathrm{K})$ and arranged as shown in Fig. 2. Here, A, B, C and D are reduced from the basic shape in the diagonal direction by up to $2 \%$, and $\mathrm{E}, \mathrm{F}$, $\mathrm{G}, \mathrm{H}, \mathrm{I}, \mathrm{J}$ and $\mathrm{K}$ are parts of A-D cut along different lines. We fabricated about ten too many for majority types (A, B, C) and selected mirrors with $R_{c}$ ranging between 4498 and $4502 \mathrm{~mm}$. The periphery region was patched by $\mathrm{E}-\mathrm{K}$ type
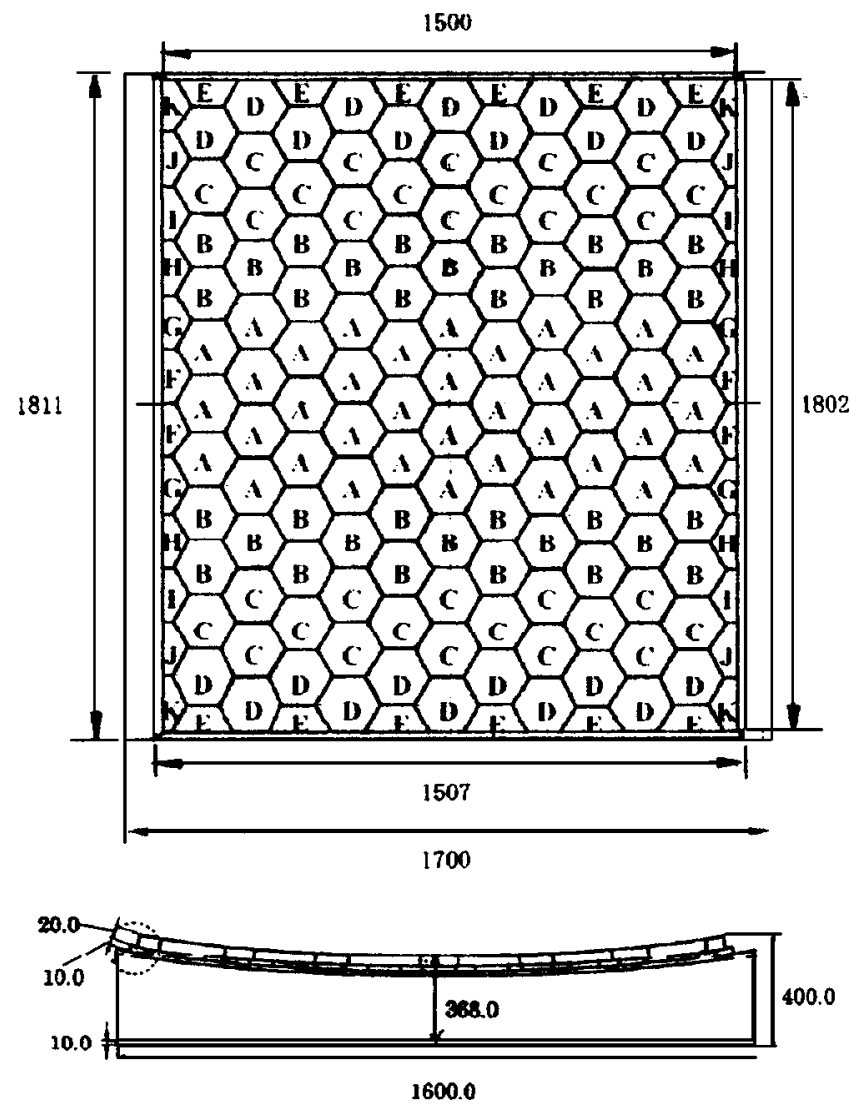

FIG. 2. Arrangement of various kinds of mirrors.

mirrors. The surface of the mirrors was coated with gold, which has the highest reflectivity in the red-to-near-infrared region.

\section{ASSEMBLING AND ADJUSTMENT}

The main components are the framework, B planes, and segment mirrors (Fig. 1), which were assembled as follows. (1) The honeycomb framework was assembled in an optics company. (2) Setting the honeycomb frame with the hollow side up, $138 \mathrm{~B}$ plates were fixed to the framework by screws. (3) An A-type mirror (M1) was bonded to a B plate around the center. (4) Six A-type mirrors (M2-M7) were arranged around M1 with 0.3-mm-thick spacers inserted among them. (5) The M2-M7 mirrors were bonded to the corresponding B plates. (8) In this way, we extended mirrors in the radial direction, by setting appropriate-type mirrors in the appropriate direction. After all segment mirrors were put together, both sides of the shaft were put on pillow blocks that keep the mirror inclined by $30^{\circ}$ in the plane and by $11^{\circ}$ in the out of plane direction.

In the actually used position, the orientation of each mirror was fine tuned in the setup shown in Fig. 3. A tiny light source of $0.1 \mathrm{~mm}$ in diameter (a tip of an optical fiber of $0.1 \mathrm{~mm}$ in diameter and numerical aperture $\sim 0.2$ ) and a charge coupled device (CCD) plate, each being separated by $30 \mathrm{~mm}$, were arranged so that the image of M1 mirror was located at the center of the CCD. In order to enable to scan the shine spot on the mirror surface while the light-source position fixed accurately, the tip of the fiber is mounted at the 


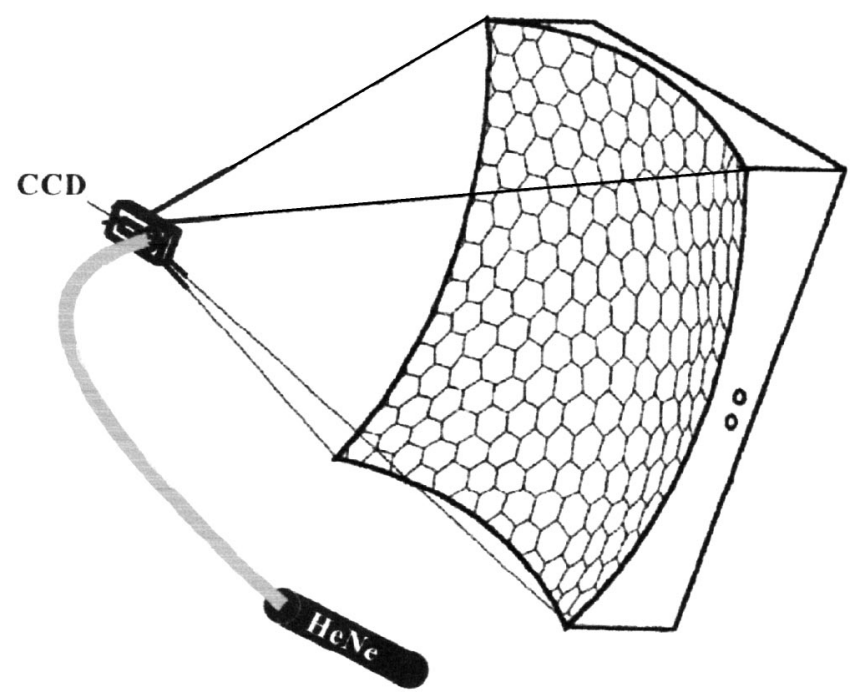

FIG. 3. Optical setup for adjusting the orientation of the segment mirrors.

center of a polystyrene ball that can rotate like that in a PC mouse. Illuminating a segment mirror to be measured, with all other mirrors being covered by paper masks, its image position on the CCD is adjusted by rotating two pushing screws so that it coincides with the common focal point on the CCD plate.

\section{PERFORMANCE AND DISCUSSION}

A surface accuracy test based on the Newton ring method indicated that the segment mirrors before bonding to the B plate are better than $\lambda / 2$ for all types of mirrors. If this surface quality is preserved after bonding, the image's size and shape should be similar to the fiber tip. Contrary to this expectation, the majority of the formed images have a diameter larger than $0.4 \mathrm{~mm}$. Moreover, some of them were highly elongated. We speculate that the segment mirrors deformed while the epoxy was curing, although a possibility that the images were defocused by a rather soft optical setup cannot be removed completely. Just after the assembly, the image size formed by all segment mirrors was around $0.6 \mathrm{~mm}$ in diameter. The image size measured about 1 year after the assembly showed appreciable growth and deformation like the Andromeda nebula. After a readjustment, the image size grew only moderately. Probably, the surfaces bonding together crept in the epoxy layer before it hardened completely. The image size after 5 years operation (see Fig. 4) was within less than $1 \mathrm{~mm}$ in diameter, which is still small enough to focus the scattered light from the laser beam less

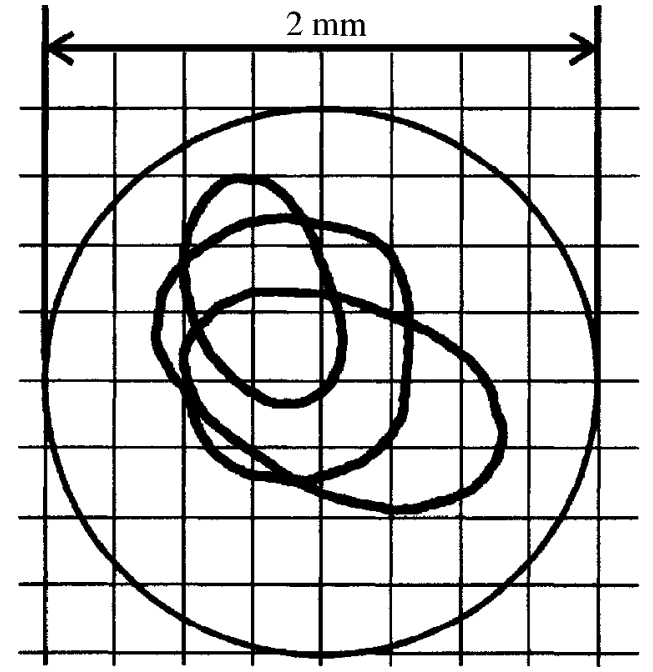

FIG. 4. Images of a $0.1 \mathrm{~mm}$ core fiber formed by several segment mirrors observed five years after the final adjustment.

than $3 \mathrm{~mm}$ in diameter onto the tip of 2-mm-diam optical fiber cut at the Brewster angel. Several mirrors' images, however, deviate largely from the common focal point, the reason for which is under examination. Loss of surface reflectivity is another concern in view of keeping the light collection capability high. After 5 years usage, the surface became somewhat misty in the visible region. We tried to softly wipe off the substance adhering to the surface by using detergent, but because of tenderness of the gold coat we could not remove it completely. A rough reflectivity measurement showed, however, that the reflectivity drop in the red-to-near-infrared region is negligible.

The lessons we have learned through this work would be helpful for those who have the intention of building similar mirrors for a variety of applications. (1) The number of the segment mirrors should be smaller than the present 138; it took us too much time for assembling and adjustment. (2) We should have been more careful in bonding the mirrors and the B plates; we should bond at three spots rather than on the whole area. (3) The mirror surface should have been coated with Al plus MgF2 rather than gold.

\section{ACKNOWLEDGMENT}

The authors acknowledge P. R. Hall of Precision-Optical Engineering for suggesting the idea of a composite mirror.

\footnotetext{
${ }^{1}$ M. Fujiwara et al., Fusion Energy 15, 7 (1996).

${ }^{2}$ K. Narihara, I. Yamada, H. Hayashi, and K. Yamauchi, Rev. Sci. Instrum. 72, 1122 (2001)
} 